\title{
Opinión del Comité Consultivo de Inmunizaciones Sociedad Chilena de Infectología. Vacuna neumocóccica conjugada en niños y la emergencia de serotipo 19A
}

\author{
Marcela Potin, Alberto Fica, Jan Wilhem, Jaime Cerda, Lily Contreras, \\ Carola Escobar, Gabriela Moreno, Alma Muñoz y Liliana Véliz
}

Conflictos de interés: L. Véliz y $\mathrm{M}$ Potin han recibido honorarios por charlas en reuniones científicas organizadas por Merck Sharp \& Dohme (MSD), L. Contreras recibió ayuda por parte de MSD para asistir a un Congreso científico, A. Muñoz ha recibido honorarios por asesorías a Pfizer y J. Wilhem ayuda por parte de Pfizer para asistir a un Congreso y honorarios por parte de GSK.

Recibido: 5 de mayo de 2016

Correspondencia a: Marcela Potin mpotin@med.puc.c

\author{
Statement of the Advisory Immunization Committee of the Chilean Society of \\ Infectious Diseases on the emergence of serotype 19A pneumococcal infection and \\ the use of pneumococcal conjugated vaccine in Chilean children
}

Inclusion of the 10-valent pneumococcal conjugated vaccine (PCV10) in the Chilean infant vaccination Program in 2011 was followed by a reduction of hospital admissions and pneumonia-related deaths in this age group. However, a progressive increase of serotype 19A pneumococcal isolates (not included in PCV10) has been observed. According to the analysis of pneumococcal strains performed by the national reference laboratory of the Institute of Public Health as part of a national surveillance on invasive pneumococcal infections, the relative proportion of serotype $19 \mathrm{~A}$ isolates increased from $\leq 5 \%$ before 2010 to $12-23 \%$ in years $2014-2015$. Serotype $19 \mathrm{~A}$ represented $4-8 \%$ of the isolates in the pre-vaccine era among children less than 2 years, increasing to $25 \%$ during 2014. This increase has been documented in two-thirds of the national territory. Among children $\leq 5$ years of age, $25 \%$ of $19 \mathrm{~A}$ serotype isolates from non-meningeal infections were penicillin resistant wheras from meningeal infections near $100 \%$ were penicillin resistant. Genetic analysis indicates that $48 \%$ of these $19 \mathrm{~A}$ strains belong to clonal complex 320, recognized for its pandemic potential and high antimicrobial resistance. Among children, most invasive infections secondary to serotype 19A have occurred in patients fully vaccinated with PCV10. These epidemiological changes indicate an increase in invasive pneumococcal infections by serotype 19A in Chile and the need to control this problem by changing the current PCV10 for the PCV13 vaccine containing serotype 19A.

Key words: Advisory committee; pneumococcal infections; Streptococcus pneumoniae serotype 19A; child; infant; 10 valent pneumococcal vaccine; 13 valent pneumococcal vaccine.

Palabras clave: Comité asesor; infecciones neumocócicas; Streptococcus pneumoniae serotipo 19A; niños; lactantes; vacuna neumocócica 10 valente, vacuna neumocócica 13 valente.

\section{Introducción}

L a enfermedad neumocóccica invasora (ENI) es un problema de salud pública mundial. Las tasas de ENI en Chile según los datos de vigilancia en la década pasada, alcanzaban a 56 casos por 100.000 en el grupo de menores de 3 años $^{1}$ y 111 a 288 casos por 100.000 bajo 2 años ${ }^{2}$. Además de su alta frecuencia, la letalidad en casos de sepsis en lactantes bordea $50 \%$ y en eventos de meningitis, $14 \%{ }^{1}$.

Para disminuir el riesgo de estas infecciones, se introdujo en Chile el año 2011 la vacunación programática de lactantes con una vacuna conjugada polivalente antineumocóccica.

En el mercado chileno se dispone actualmente de dos vacunas anti-neumocóccicas conjugadas que difieren en la cantidad de serotipos (ST) incluidos y en las proteínas a los que éstos se conjugan. Una de ellas contiene 10 serotipos $(1,4,5,6 \mathrm{~B}, 7 \mathrm{~F}, 9 \mathrm{~V}, 14,18 \mathrm{C}, 19 \mathrm{~F}$ y $23 \mathrm{~F}$;
Synflorix ${ }^{\circledR}$, Glaxo SmithKline, PCV10) y la otra 13 serotipos (Prevenar 13®, Pfizer, PCV13) que incluye los 10 anteriores más los serotipos 3, 6A y 19A.

La vacuna incorporada el 2011 al Programa Nacional de Inmunizaciones (PNI) corresponde a la vacuna PCV10 que se aplicó inicialmente en tres dosis, a los 2, 4 y 6 meses de edad con un refuerzo a los 12 meses de edad (esquema denominado 3+1). El año 2012 se modificó el esquema, administrándose desde entonces sólo dos dosis, a los 2 y 4 meses de edad, manteniendo el refuerzo a los 12 meses (esquema $2+1$ ).

\section{Impacto mundial de las vacunas antineumocóccicas conjugadas}

Los países que han introducido estas vacunas en niños han observado una notable reducción en las tasas de ENI en el grupo vacunado por los ST contenidos en la vacuna. 
En países que alternativamente han usado PCV13, se observa adicionalmente un efecto indirecto de reducción neta de las tasas de ENI en adultos mayores (efecto rebaño); sin embargo, esto no es claro para países que usan PCV10 ya que si bien se reducen los ST incluidos en la vacuna, aumentan aquellos no incluidos, lo que mantiene las tasas de enfermedad. Esto es evidente en Finlandia en adultos sobre 65 años y en Brasil en adultos sobre 40 años ${ }^{3,4}$.

Los países que han usado vacunas de 7 (no disponible actualmente) o $10 \mathrm{ST}$, han evidenciado una emergencia de infecciones por el serotipo 19A no incluido en estas dos vacunas, lo que reviste especial importancia dado su perfil de resistencia. Ello ha llevado a que países como Nueva Zelandia, regiones de Canadá como Quebec, México y Perú, hayan decidido cambiar su vacuna pediátrica hacia la PCV13.

\section{Impacto en Chile}

La información sobre los ST circulantes de $S$. pneumoniae en Chile es obtenida a través de la derivación de cepas hacia el Laboratorio de Referencia Nacional del Instituto de Salud Pública (ISP). Hasta el año 2007, esta derivación involucraba mayormente la Región Metropolitana y desde entonces, todas las regiones del país deben colaborar con la vigilancia enviando las cepas de $S$. pneumoniae identificadas en infecciones invasoras. Se reconoce que la vigilancia no es óptima ya que depende de variables como la posibilidad o costumbre de los médicos de realizar hemocultivos en niños con fiebre $\sin$ foco, de la dotación de insumos óptimos en los laboratorios del país y del apropiado traslado de las cepas al ISP. No obstante, en la actualidad prácticamente $100 \%$ de los aislados de $S$. pneumoniae causantes de ENI logran ser tipificados ${ }^{5}$.

Al comparar el período pre vacuna (años 2007-2010) con el post vacuna (2012-2015) se observa una reducción general de $21,7 \%$ del total de cepas confirmadas como $S$. pneumoniae, provenientes de pacientes con ENI. Esta reducción alcanza a $61,9 \%$ en niños bajo 2 años de edad $^{6}$. Por otra parte, en un estudio caso control en niños vacunados con PCV10 se pudo estimar una efectividad de la vacuna de $20,7 \%$ para prevenir eventos de neumonía y de $71,5 \%$ para muertes por neumonía ${ }^{7}$.

Luego de la introducción de la vacuna, en personas mayores de 60 años el número de casos anuales de ENI notificados al ISP no se ha modificado. Si bien se observa una reducción de los aislados de ST incluidos en la vacuna, existe un incremento de los que no están incluidos, el 19A y el 3, lo que compensa y finalmente reduce en forma importante el efecto rebaño de la vacuna.

Cambios en los serotipos luego de la introducción de PCV10. Los principales cambios en la población general se observaron en los ST 1, 14 y 5. Así, los aislados del serotipo
1 disminuyeron en $74,8 \%$, el serotipo 14 en $60,8 \%$ y el serotipo 5 en $80,6 \%$ en relación al período prevacuna ${ }^{6}$. En contraste, el serotipo 19A, no incluido en PCV10 aumentó en 270 a $350 \%$ en la población general, ya que antes de la vacunación su frecuencia relativa era 3,3 a 4,8\% del total de cepas de ENI, llegando a $12 \%$ el año 2014 y a $13 \%$ el $2015^{6}$. En niños bajo 2 años de edad, estos cambios han sido incluso más notorios. Así, de una frecuencia promedio de 5,75\% (rango 4 a $8 \%$ ) en la era pre vacuna, este serotipo aumentó a $25 \%$ el año 2014 y a 19\% el $2015^{6}$. Además, en 10 de las 15 regiones del país se han observado incrementos de aislados pertenecientes al serotipo 19A6 .

Por otro lado el análisis de los pacientes bajo 5 años afectados por ENI, entre enero y mayo del año 2015, indica un incremento de las cepas pertenecientes al complejo $6 \mathrm{ABC}$, el que junto a 19A dan cuenta de $40 \%$ de los casos de ENI en niños en ese período (comunicación personal de Cecilia González, Programa Nacional de Inmunizaciones, MINSAL, Chile).

Un estudio focalizado en los casos de infecciones en niños por el serotipo 19A durante los primeros meses del año 2015, indica que de los 23 casos afectados, la mayor parte tenía el esquema de vacunación con PCV10 al día ( 19 de 22 con datos disponibles) y que 22 niños tenían al menos dos dosis de vacuna ${ }^{8}$. Ello permite descartar que la emergencia del serotipo 19A ocurra en niños no vacunados y sugiere en cambio que PCV10 no está generando protección cruzada contra este serotipo.

\section{Relevancia de la emergencia del serotipo 19A}

El comportamiento del serotipo 19A es preocupante pues, el estudio molecular realizado por el ISP con cepas de $S$. pneumoniae del año 2015 confirmó que 48\% de las cepas del serotipo 19A circulante corresponde al clon 320, de carácter pandémico, y que se asocia a multi-resistencia ${ }^{9}$. Así, en pacientes bajo 5 años de edad, el serotipo $19 \mathrm{~A}$ muestra $25 \%$ de resistencia a penicilina para aislados extrameníngeos y casi $100 \%$ para los de meningitis. Estos antecedentes refuerzan el concepto que la vacuna PCV10 no sólo se asocia a la emergencia de un nuevo serotipo, sino que este cambio trae consigo un riesgo de fracaso en la terapia antimicrobiana y un aumento de los costos del tratamiento de las ENI al requerir el uso de cefalosporinas de tercera generación, lo que a su vez contribuye a incrementar la resistencia antimicrobiana.

\section{En resumen}

Luego de cinco años de introducida la vacuna PCV10 en lactantes en Chile, se observa una notable reducción del numero casos de ENI en niños bajo 5 años de edad, asi como un impacto moderado en las hospitalizaciones 
y muertes por neumonía probablemente bacteriana en este grupo etario.

Sin embargo, en niños sobre 5 años y adultos se observa un aumento en el número de cepas totales de $S$. pneumoniae provenientes de ENI derivadas al ISP, ya que si bien los ST incluidos en la vacuna descienden, se observa un incremento de los ST no contenidos. Esto es especialmente relevante en el adulto mayor. Los estudios realizados con PCV13 en niños en otros países muestran una notable reduccción de la carga de enfermedad en adultos, efecto que sería de enorme utilidad en nuestro país.

Adicionalmente el incremento del serotipo 19A en números absolutos y relativos resulta preocupante, en especial porque se asocia al complejo clonal 320 de carácter pandémico y de resistencia a antimicrobianos.

Los datos disponibles sugieren que la vacuna en uso no evidencia una protección cruzada contra el serotipo 19A pues los niños vacunados con PCV10 están presentando infecciones invasoras por este serotipo.

Por ello nos parece necesario reemplazar la actual vacuna PCV10 por la vacuna PCV13 en niños. Esta recomendación de cambio ya ha sido adoptada por otros países y en nuestro caso surge en respuesta al análisis crítico de la información obtenida a través de la vigilancia disponible.

Es menester, además, mejorar la vigilancia de ENI, integrando los datos de laboratorio con la información clínica de los casos de ENI.

\section{Resumen}

La incorporación de la vacuna conjugada antineumocóccica 10 valente (PCV10) en lactantes en Chile el año 2011 ha permitido reducir las hospitalizaciones y muertes por neumonía en este grupo etario. Sin embargo, se ha observado desde entonces un aumento progresivo de los aislados de Streptococcus pneumoniae del serotipo 19A no incluido en la vacuna en uso (de $\leq 5 \%$ del total de cepas recibidas en el Laboratorio de Referencia Nacional del Instituto de Salud Pública para vigilancia de infecciones invasores causadas por $S$. pneumoniae hasta el año 2010, a $12-23 \%$ en los años 2014-2015). En lactantes, el serotipo 19A representaba 4 a $8 \%$ de los aislados en la era pre vacuna, porcentaje que se incrementa a $25 \%$ el 2014 . Este aumento ha ocurrido en dos terceras partes de las regiones administrativas del país. Cepas del serotipo 19A de pacientes menores de 5 años, muestran $25 \%$ de resistencia a penicilina para aislados extra-meníngeos y casi $100 \%$ para aislados de meningitis. El análisis genético de las cepas del serotipo 19A ha demostrado que $48 \%$ pertenecen al complejo clonal 320 de carácter pandémico y asociado a resistencia antimicrobiana. Además, casi todas las infecciones invasoras por serotipo 19A en niños se han dado en pacientes con esquema completo de vacunación PCV10. Los cambios epidemiológicos presentados indican la emergencia de infecciones invasoras por el serotipo 19A y la necesidad de controlar este problema con el cambio de la vacuna PCV10 a la vacuna PCV13 que contiene el serotipo 19A.

\section{Referencias bibliográficas}

1.- Lagos R, Muñoz A, Valenzuela M T, Heitmann I, Levine M M. Population-based surveillance for hospitalized and ambulatory pediatric invasive pneumococcal disease in Santiago, Chile, 1994-2007. Pediatr Infect Dis J 2002; 21: 1115-23.

2.- Abarca K, Vergara R. Tassara E, Ibáñez I, García C, Potin M. Infección neumocócica invasora y neumonía condensante en lactantes, un año de vigilancia en tres centros hospitalarios chilenos, Rev Chilena Infectol 2008; 25: 97-103

3.- National Institute for Health and Welfare. Incidence of pneumococcal disease in Finland. Disponible en: https://www.thl. fi/web/thlfi-en/research-and-expertwork/ projects-and-programmes/monitoring-thepopulation-effectiveness-of-pneumococcalconjugate-vaccination-in-the-finnish-nationalvaccination-programme/incidence-of-invasivepneumococcal-disease-in-finland (accedido el 2 de mayo de 2016).

4.- Andrade A L, Minamisava R, Policena G, Cristo E B, Domingues C M, de Cunto Brandileone B C, et al. Evaluating the impact of PCV 10 on invasive pneumococcal disease in Brazil: a time series analysis. Hum Vacc Immunother 2016; 12: 285-92.

5.- Vigilancia de laboratorio de Streptococcus pneumoniae procedente de enfermedad invasora. Chile, 2007-2015. Boletín de Vigilancia de Laboratorio Instituto de Salud Pública de Chile. Julio de 2015, vol 5, Nº 7 , julio 2015. Disponible en: http://www.ispch.cl/ sites/default/files/Bolet $\%$ C3\%ADn $\% 20 \mathrm{de} \% 20$ Vigilancia\%20de $\% 20$ Laboratorio $\% 20 \mathrm{de} \% 20$ Streptococcus\%20pneumoniae.pdf. (Accedido el 2 de mayo de 2016)

6.- Comportamiento de Streptococcus pneumoniae serotipos 3 y 19 A en Chile, años 2007-2015, Boletín de Vigilancia de Laboratorio Instituto de Salud Pública de Chile. 2016, vol 6, No 2, febrero de 2016. Disponible en: http://www.ispch.cl/sites/ default/files/Boletin $\% 20$ S.pneumoniae $\% 20$ 19A\%20y\%203.pdf (accedido el 2 de mayo de 2016).

7.- Díaz J, Terrazas S, Bierrenbach A L, Toscano C M, Alencar G P, Álvarez A, et al. Effectiveness of the 10-valent pneumococcal conjugate vaccine (PCV-10) in children in Chile: a nested case-control study using nationwide pneumonia morbidity and mortality surveillance data. PLoS ONE 2016; 11: e0153141.

8.- Potin M, Díaz J, Hormazábal J C, Pidal P, González C, Avendaño M, et al. Comportamiento del serotipo 19 A de Streptococcus pneumoniae en niños bajo los 5 años en la era post introduccción de vacuna conjugada. Libro de resúmenes del XXXII Congreso Chileno de Infectología 2015, Viña del Mar; Chile, CO. Disponible en: http://www.sochinf.cl/portal/templates/ sochinf2008/documentos/2015/Libro congreso_2015.pdf (accedido el 2 de mayo de 2016)

9.- Alarcón P, Hormazábal J C, Araya P, Hormazábal J, Puentes R, Díaz J, et al. Vigilancia de laboratorio para Streptococcus pneumoniae proveniente de enfermedad invasora, serotipos circulantes en Chile. Instituto de Salud Pública (ISO), Chile Libro de resúmenes del XXXII Congreso Chileno de Infectología 2015, Viña del Mar; Chile Com oral 6. Disponible en: http://www.sochinf.cl/ portal/templates/sochinf2008/documentos/2015/ Libro congreso 2015.pdf (accedido el 2 de mayo de 2016). 